\title{
Glibenclamide elicits endoplasmic reticulum stress and myokine expression in the L6 skeletal muscle cells
}

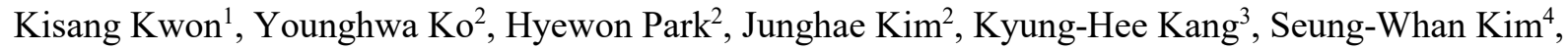 \\ $\mathrm{O}-\mathrm{Yu} \mathrm{Kwon}{ }^{2 *}$ \\ ${ }^{1}$ Department of Clinical Laboratory Science, Wonkwang Health Science University, Iksan 54538, \\ Korea \\ ${ }^{2}$ Department of Anatomy \& Cell Biology, College of Medicine, Chungnam National University, \\ Daejeon 35015, Korea \\ ${ }^{3}$ Department of Dental Hygiene, College of Medical Science, Konyang University, Daejeon 35365, \\ Korea \\ ${ }^{4}$ Department of Emergency Medicine, College of Medicine, Chungnam National University, Daejeon \\ 35015, Korea \\ *Corresponding author: oykwon@cnu.ac.kr
}

Received: May 30, 2021. Revised: December 15, 2021. Accepted: January 14, 2022. Published: January 18, 2022.

\begin{abstract}
Although glibenclamide is an oral hypoglycemic agent used in type 2 diabetes, skeletal muscle wasting has been reported as a side effect. To understand how to reduce this side effect, we determined whether glibenclamide induces endoplasmic reticulum (ER) stress in skeletal muscle cells and which myokine expression changes at this time. The ER chaperone genes do not show a significant change by glibenclamide, but the ER stress sensor genes are upexpressed approximately twice, and those downstream [ATF6 (activating transcription factor 6) fragmentation, eIF2 $\alpha$ (eukaryotic initiation factor-2 $\alpha$ ) phosphorylation, and XBP1 (Xbox DNA-binding protein) mRNA splicing] are activated. Additionally, the myokine gene expression was up- or downregulated by glibenclamide. These results will serve as useful data for overcoming the side effects of sarcopenia caused by glibenclamide.
\end{abstract}

Keywords-endoplasmic reticulum (ER) stress, glibenclamide, L6 skeletal muscle cells, myokines

\section{INTRODUCTION}

$\mathrm{G}$ LIBENCLAMIDE, also known as glyburide, was first synthesized in 1969 and has been used in aged patients affected by impaired glucose-induced insulin release in the United States since 1984. Presently, glibenclamide is commonly used as an antidiabetic agent in managing type 2 diabetes [1]. It inhibits $\mathrm{K}_{\text {ATP }}$ in pancreatic beta cells, thereby activating the voltage-sensitive calcium channel, which increases the intracellular calcium concentration and accelerates insulin secretion [2]. It has been reported that physiological side effects of glibenclamide include nausea, heartburn, angioedema, and hypoglycemia [3]. Several other effects under biomedical research have already been reported, such as apoptosis induction, inhibition of extracellular matrix formation, inhibition of cell growth, and increase in twitch and tetanus tension by glibenclamide [4-7]. Although another beneficial function of glibenclamide is reported to reduce edema-hemorrhage in traumatic brain injury and apoptosis induction in cancer cells, the most interesting side effect is skeletal muscle wasting associated with type 2 diabetes [8]. As many aged people have already been exposed to sarcopenia and diabetes, glibenclamide is used as an oral drug for treating type 2 diabetes. Some in vitro studies reported that muscle atrophy associated with type 2 diabetes was a side effect of glibenclamide; it was also demonstrated that $\mathrm{K}_{\mathrm{ATP}}$ mainly acts as a molecular sensor in skeletal muscle atrophy [9]. The International Working Group on Sarcopenia defined sarcopenia as "age-associated loss of skeletal muscle mass and function," in 2011 [10] WMC and the European Working Group on Sarcopenia in Older People declared it as "a syndrome characterized by progressive and generalized loss of skeletal muscle mass and strength with a risk of adventure outcomes, such as physical disability, poor quality of life, and death" in 2019 [11].

The endoplasmic reticulum (ER) of eukaryotic cells is a critical site for posttranslational modification, including protein folding and assembly. ER lumen has many ER chaperones 
[binding immunoglobulin protein $(\mathrm{BiP})$, calnexin, protein disulfide isomerase (PDI), and ER protein 29 (ERp29)] responsible for various functions. Furthermore, three types of ER stress sensors [inositol requiring enzyme 1 (IRE1), PKR like ER kinase (PERK), and activating transcription factor 6 (ATF6)] are activated [12]. ER stress in mammalian cells is triggered by the dissociation of $\mathrm{BiP}$ due to stress transducers, such as PERK, ATF6, and IRE1. As ER stress signal and other intercellular signals are closely correlated, it is frequently checked up during the initial stage of developing new drugs, in addition to drug efficacy and side effect verification. In this study, we tested whether glibenclamide induces ER stress signal in L6 skeletal muscle cells. By understanding the ER stress signal using glibenclamide in muscle cells, we expect to provide a better understanding of how to reduce the side effects of sarcopenia induction by glibenclamide.

\section{MATERIALS AND METHODS}

L6 rat myoblast-derived cells were maintained at $37^{\circ} \mathrm{C}$ with $5 \% \mathrm{CO} 2$ in collagen coated flasks containing DMEM supplemented with $10 \% \quad$ FBS and $100 \quad \mu \mathrm{g} / \mathrm{ml}$ penicillin-streptomycin antibiotic [13]. When the cells showed $>90 \%$ confluence, they were maintained with no changes in culture medium for 2-3 days more to induce myotube formation. Glibenclamide $\left(\mathrm{C}_{23} \mathrm{H}_{28} \mathrm{ClN}_{3} \mathrm{O}_{5} \mathrm{~S} ; 494.004 \mathrm{~g} / \mathrm{mol}\right)$ purified to $\geq 99 \%$ (HPLC) was purchased from Sigma Aldrich. Total RNA was extracted from L6 cells using the SV Total RNA Isolation System (Promega, Madison, WI, USA) and quantified using the NanoDrop Lite UV-spectrophotometer (Thermo Scientific, Waltham, MA, USA).

The mRNA in the samples was reverse-transcribed using the SuperscriptII ${ }^{\mathrm{TM}}$ First Strand Kit (Invitrogen Carlsbad, CA, USA). RT PCR conditions included 30 cycles as follows: $94^{\circ} \mathrm{C}$ for $30 \mathrm{~s}, 58^{\circ} \mathrm{C}$ for $30 \mathrm{~s}$, and $72^{\circ} \mathrm{C}$ for $1 \mathrm{~min}(10 \mathrm{~min}$ in the final cycle) using primers with Taq DNA polymerase. The RT-PCR bands were quantitatively analyzed using the ImageJ program of NIH. The RT PCR primers supplied by Bioneer Co. (Daejeon, Korea) and used in this study are listed in Table 1 (BiP; binding immunoglobulin protein, Canx; calnexin, PDI; protein disulfide isomerase, ERp29; ER protein 29, PERK; protein kinase RNA-like ER kinase, ATF6; activating transcription factor 6, IRE1; inositol requiring protein 1, XBP1; X-box binding protein-1, LC3a; autophagy marker light chain 3a, Beclin, Bax; BCL2 associated X, Bak, BCL-2 killer, Bcl-2; B-cell lymphoma 2, Bid; BH3-interacting domain death agonist, MyoD1; myogenic differentiation 1, MyoG; myogenin, Vfgfa; vascular endothelial growth factor a, BMP7; bone morphogenetic protein 7, IL-6; interleukin-6, IL15 interleukin-15, Apln; apelin, Sesn1; sestrin 1, SDF1; stromal cell-derived factor 1, SPARC; secreted protein acidic and rich in cysteine, Visfatin, Musclin, Dcn; decorin, PPIA; peptidylprolyl isomerase A). The size difference between spliced and unspliced XBP1 mRNA was measured. Total RNA was reverse-transcribed, and double strand cDNA was synthesized by PCR using specific sense and anti-sense primers described in Table 1. The amplified cDNA was treated with PstI, and the resulting products were analyzed by electrophoresis in a $2.5 \%$ agarose gel. All undescribed chemicals were purchased from Sigma Aldrich.

Western blotting was performed briefly with modifications and L6 cells were lysed using RIPA buffer $(25 \mathrm{mM}$ Tris- $\mathrm{HCl} \mathrm{pH}$ $7.6,150 \mathrm{mM} \mathrm{NaCl}, 1 \% \mathrm{NP}-40,1 \%$ sodium deoxycholate, $0.1 \%$ SDS, and $1 \mathrm{mM}$ phenylmethylsulfonyl fluoride). The total protein concentration was estimated using Pierce BCA protein assay Kits (Thermo Fisher Scientific, Cleveland, OH, USA). Proteins were separated using SDS-PAGE and transferred to a nitrocellulose membrane. The resulting membrane was incubated with primary antibodies overnight at $4^{\circ} \mathrm{C}$, followed by incubation with secondary antibodies. It was developed using an enhanced chemiluminescence Western blotting detection system Kit (Amersham, Sweden) according to the manufacturer's instructions. Anti-ATF6, anti-eIF2 $\alpha$, anti-eIF2 $\alpha-P$, and goat anti-actin antibodies were obtained from Santa Cruz Biotechnology (Santa Cruz, CA, USA).

\section{RESULTS AND DISCUSSION}

We tested the effect of glibenclamide in inducing the expression of ER chaperones (BiP, Calnexin, PDI, and ERp29) and ER stress sensors (PERK, ATF6, and IRE1). BiP is an ER chaperone located in the ER lumen, which binds newly-synthesized proteins during translation. However, it is bound to misfolded proteins and ER stress sensors under ER stress conditions. Calnexin plays a vital role in the proofreading of misfolded glycoproteins in the ER lumen. PDI is also located in the ER lumen, which catalyzes the formation and breakage of disulfide bonds between cysteine residues within folding proteins. ERp29 is highly homologous with PDI and is involved in processing secretory proteins (12)

The expression of $\mathrm{BiP}$ and ERp29 was approximately 2.5 and 4 times higher than ER stress-inducible tunicamycin, respectively, and only approximately 1.5 times higher than PDI and ERp29 by glibenclamide. The result of Fig. 1A suggests that glibenclamide does not induce remarkable expression of ER chaperones. PERK is a type I transmembrane protein kinase that inhibits the overall protein translational process by phosphorylation of eIF2 by ER stress. ATF6 is an ER membrane-type II protein that produces ATF6c transcription factor cleaved by S1P and S2P upon ER stress. IRE1 is autophosphorylated by ER stress to induce XBP1 mRNA splicing. The expression of three ER stress sensors by glibenclamide was almost the same as that by tunicamycin (Fig. 1B result). The result of Fig. 1B suggests that glibenclamide regulates the expression of ER stress sensors at almost the same level as tunicamycin. 
A

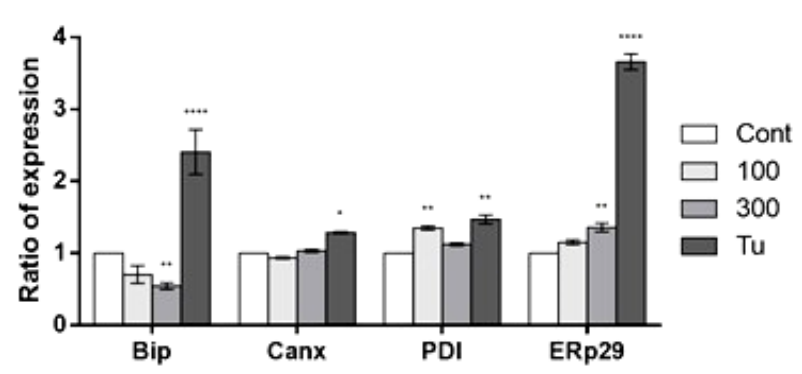

C

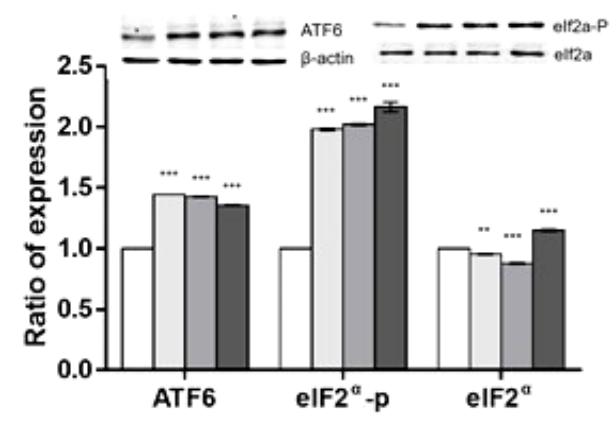

B

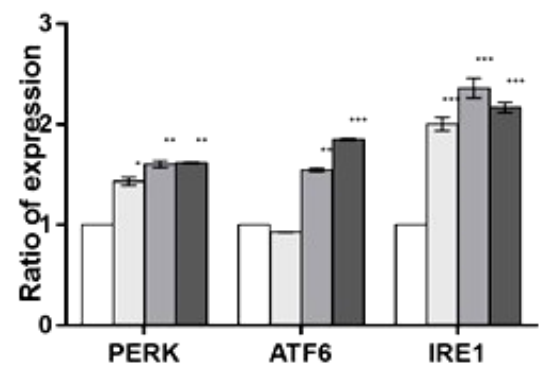

D

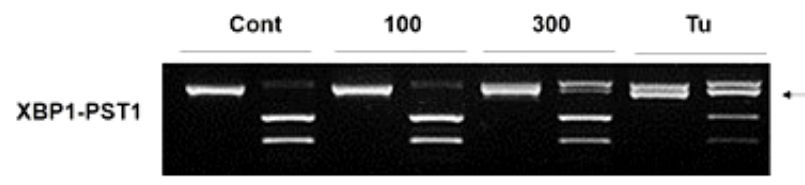

Fig. 1: The effect of glibenclamide on ER stress. (A and B) L6 cells were treated with glibenclamide (100 and $300 \mu \mathrm{g} / \mathrm{ml})$ and tunicamycin $(25 \mathrm{ng} / \mathrm{mL})$ for $24 \mathrm{~h}$. Gene expression of both ER chaperones (BiP, Canx, PDI, and ERp29) and ER stress sensors (PERK, ATF6, and IRE1) was demonstrated by RT-PCR. Tu, tumicamycin; Canx, Calnexin. (C) Western blotting was performed using an anti-ATF6 monoclonal antibody, anti-eIF2- $\alpha$ antibody, and eIF2- $\alpha$-P antibody against cells treated with different doses of glibenclamide (100 and $300 \mu \mathrm{g} / \mathrm{ml}$ ) for $24 \mathrm{~h}$. (D) RT-PCR was performed for the XBP1 mRNAs. Pst further digested the resulting PCR products to reveal a lost restriction site following XBP1 splicing under ER stress. Digested XBP1 cDNA products were revealed on a 2\% agarose gel. Unspliced XBP1 mRNA is indicated only by an arrow. Statistical significance between multiple groups: one-way analysis of variance test. Analysis was performed using GraphPad Prism v.6 software (GraphPad Software Inc.). Mean \pm SEM. $\mathrm{n}=6 . * \mathrm{p}<0.05 * * \mathrm{p}<0.005 * * * \mathrm{p}<0.001 * * * * \mathrm{p}<0.0001$

Three ER stress sensors regulate the expression of various genes related to protein homeostasis by activating specific signaling pathways and downstream factors. ATF6 is cleaved at the cytoplasmic N-terminus, a transcription factor of ATF6c with a bZIP domain that increases the transcription of chaperone molecules and ER-associated degradation-related genes. eIF2- $\alpha$ phosphorylation inhibits the activity of eIF2B, which regulates GDP-GTP exchange and interferes with the formation of complexes required for protein translation, thereby inhibiting overall protein biosynthesis. IRE1 activates the endonuclease domains, which cleave XBP mRNA, generating an activated form of the XBP1 mRNA for XBP1 protein. Next, we tested the downstream of three ER stress sensor signals, such as ATF6 fragmentation, eIF2- $\alpha$ phosphorylation, and XBP1 mRNA splicing [14]. The ATF6c generated by fragmenting ATF6 by glibenclamide was about 1.5 times higher than its control, and eIF2 phosphorylation was about twice as high as the control (Fig. 1C). As observed regarding the ER stress-inducible drug tunicamycin, unspliced XBP1 mRNA by glibenclamide $(300 \mu \mathrm{g} / \mathrm{ml})$ treatment is shown by an arrow (Fig. 1D). Although ER chaperone expression was not induced by glibenclamide (Fig. 1A result), glibenclamide induces the expression of ER stress sensors and activates those downstream (Fig. 1B-D).

Following, we observed that glibenclamide induces gene regulation associated with autophagy, apoptosis, myogenesis, and myokines. Autophagy is a mechanism by which cells break down and recycle unnecessary or nonfunctional cell components for survival, closely related to cell death and disease induction [15]. Apoptosis is programmed cell death observed in multicellular organisms, and cells die due to cell shape and internal biochemical changes [16]. Myogenesis is the differentiation from somatic stem cells to muscle progenitor cells and myofiers in adults [17]. Skeletal muscle is an important tissue that maintains the function of our body, but recent studies have reported that secreting bioactive substances similar to various hormones secreted by endocrine organs. They are called myokines that is a compound word of myocyte, which means muscle cells, and cytokine, a kind of small hormone, which means cytokine secreted from the skeletal muscle $[18$, 19].

Both LC and Beclin genes were investigated after the 
glibenclamide treatment in L6 cells to determine whether autophagy is induced by glibenclamide. As seen in the results of
Fig. 2A, no remarkable differentiated expression was observed compared with the control. However, the expression
A

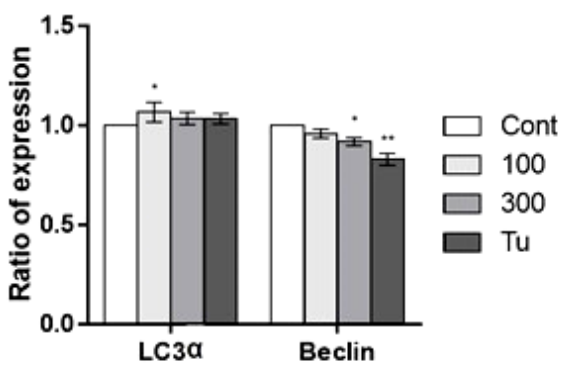

C

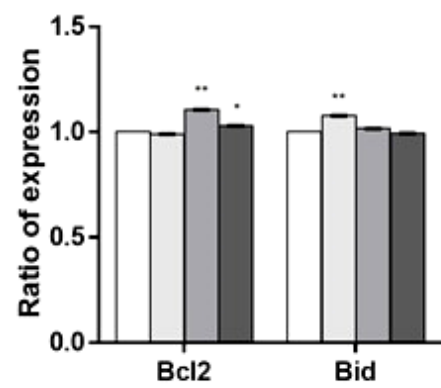

B

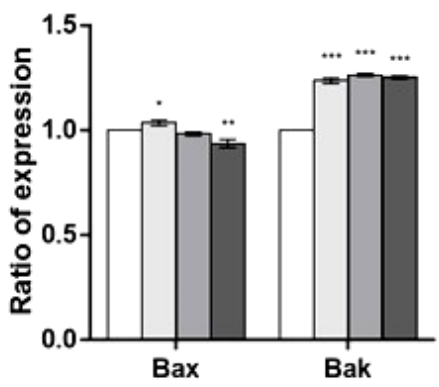

D

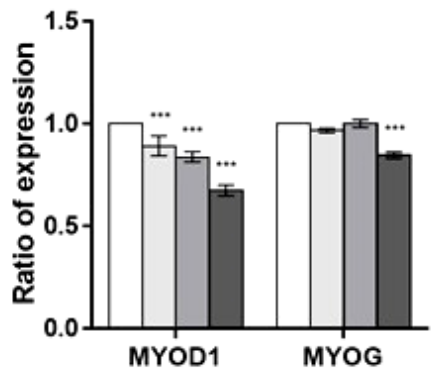

E

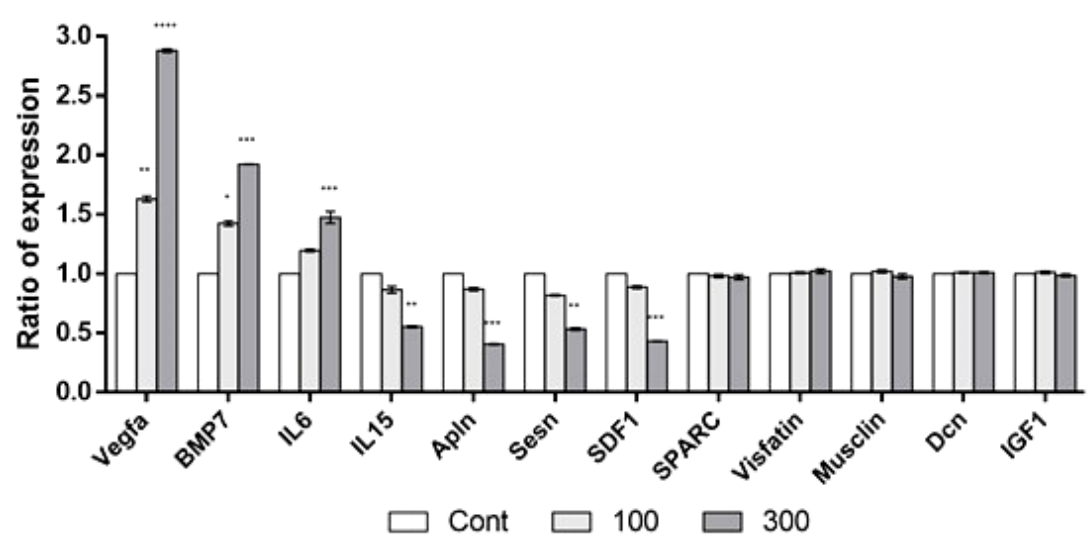

Fig. 2: The effect of glibenclamide on cell death and myokine expression. (A, B, and C) L6 cells were exposed to glibenclamide at 100 and $300 \mu \mathrm{g} / \mathrm{ml}$ for $24 \mathrm{~h}$ to confirm apoptosis and autophagy. Gene expression was measured using RT-PCR primer. LC3a, microtubule-associated protein 1A/1B-light chain 3a; Bax, Bcl2 associated X; Bak, Bcl2 Antagonist/Killer 1; Bcl2, B-cell lymphoma 2; Bid, BH3-only Bcl-2 family member. (D) The effect of glibenclamide on gene expression of myogenesis factors. MyoD1, myogenic differentiation 1; MyoG, myogenin. (E) The effect of glibenclamide on gene expression of myokines. VEGFA, vascular endothelial growth factor-a; BMP7, bone morphogenetic protein 7; IL6, interleukin 6; IL15, interleukin 15; Apln, apelin; Sesn, sestrin; SDF1, stromal cell-derived factor 1; SPARC (osteonectin), secreted protein acidic and rich in cysteine; Dcn, decorin; IGF1, insulin-like growth factor-1. Mean \pm SEM. $\mathrm{n}=6 .{ }^{*} \mathrm{p}<0.05 * * \mathrm{p}<0.005 * * * \mathrm{p}<0.001 * * * * \mathrm{p}<0.0001$. 
of apoptosis-related genes by glibenclamide and apoptosis induction (Bax and Bak) and inhibition (Bacl2 and Bid) was investigated. No significant changes in expression were observed, except for a slight increase (about 1.3 times) of the Bak gene expression related to apoptosis induction (Fig. 2B and $2 \mathrm{C}$ ). The results of Fig. 2A-C indicate that glibenclamide is not actively involved in the induction of autophagy and apoptosis; glibenclamide did not affect the degradation of the muscle cells. Moreover, the gene expression of myogenic regulatory factors of MyoD1 and MyoG was investigated to determine the effect of glibenclamide on muscle differentiation. Glibenclamide weakly suppresses the expression of the MyoD1 gene at a high concentration, but no change is observed in the case of MyoG (Fig. 2D). Finally, we tested 12 myokines to determine how glibenclamide affects myokine expression in the muscle cells (Fig. 2E). Expression patterns were divided into three: gene expression increased, descended, and unchanged. The gene expression of VEGFA was increased by about three times, BMP7 about twice, and IL6 about 1.5 times compared with the control. The gene expression reduced by half in IL15, aplin, sesn, and SDF1, and the rest showed no significant change.

In summary, although gene expression of ER chaperones (BiP, Calnexin, PDI, and ERp29) was not affected by glibenclamide, which activated the genes of ER stress sensor (PERK, ATF6, and IRE1) and those downstream, such as ATF6 fragmentation, phosphorylation of eIF2- $\alpha$, and XBP1 mRNA splicing. Furthermore, gene expression related to autophagy and apoptosis was not affected by glibenclamide. However, myokines of VEGFA, BMP7, and IL6 gene expression were selectively increased by glibenclamide, whereas myokines of IL15, aplin, sesn, and SDF1 were selectively suppressed. Even if our results can fully explain sarcopenia induction by glibenclamide, they provide an opportunity to observe the behavior of related factors in muscle cells and not harmful effects. Depolarization occurs due to increased intracellular $\mathrm{K}+$ because of KATP inhibition by glibenclamide, and a large amount of $\mathrm{Ca}++$ is introduced through the voltage-sensitive calcium channel [20-22]. In case the delicate mechanisms are resolved singularly by comprehensively considering the changes in intracellular environments caused by glibenclamide and selective expression of myokines genes, a better understanding of the solution of skeletal muscle wasting caused by glibenclamide can be expected.

\section{ACKNOWLEDGEMENTS}

This work was supported by research fund of Chungnam National University.

\section{References}

[1] D. Sola, L. Rossi, G. P. Carnevale Schianca, P. Maffioli, M. Bigliocca, R. Mella, et al. Sulfonylureas and their use in clinical practice. Arch Med Sci. 11(4): 840-848. 2015.

[2] S. Seino. ATP-sensitive potassium channels: a model of heteromultimeric potassium channel/receptor assemblies. Annu Rev Physiol. 61: 337-362. 1999.

[3] T. G. Skillman, J. M. Feldman. The pharmacology of sulfonylureas. Am J Med. 70(2): 361-372. 1981.

[4] G. Giannico, P. Cortes, M. H. Baccora, C. Hassett, D. W. Taube, J. Yee. Glibenclamide prevents increased extracellular matrix formation induced by high glucose concentration in mesangial cells. Am J Physiol Renal Physiol. 292(1): F57-65. 2007.

[5] F. Sawada, T. Inoguchi, H. Tsubouchi, S. Sasaki, M. Fujii, Y. Maeda, et al. Differential effect of sulfonylureas on production of reactive oxygen species and apoptosis in cultured pancreatic beta-cell line, MIN6. Metabolism 57(8): 1038-1045. 2008.

[6] F. Andrade, X. Trujillo, E. Sánchez-Pastor, R. Montoya-Pérez, A. Saavedra-Molina, M. Ortiz-Mesina, et al. Glibenclamide increases post-fatigue tension in slow skeletal muscle fibers of the chicken. J Comp Physiol B. 181(3): 403-412. 2011.

[7] M. Núñez, V. Medina, G. Cricco, M. Croci, C. Cocca, E. Rivera, et al. Glibenclamide inhibits cell growth by inducing $\mathrm{G} 0 / \mathrm{G} 1$ arrest in the human breast cancer cell line MDA-MB-231. BMC Pharmacol Toxicol. 14: 6. 2013.

[8] M. Cetrone, A. Mele, D. Tricarico. Effects of the antidiabetic drugs on the age-related atrophy and sarcopenia associated with diabetes type II. Curr Diabetes Rev. 10(4): 231-237. 2014.

[9] D. Tricarico, A. Mele, G. M. Camerino, R. Bottinelli, L. Brocca, A. Frigeri, et al. The KATP channel is a molecular sensor of atrophy in skeletal muscle. J Physiol. 588(Pt 5): 773-784. 2010.

[10] W. M. C. Chumlea, M. Cesari, W. J. Evans, L. Ferrucci, R. A. Fielding, M. Pahor, et al. Sarcopenia: designing phase IIB trials. J Nutr Health Aging 15(6): 450-455. 2011.

[11]A. J. Cruz-Jentoft, G. Bahat, J. Bauer, Y. Boirie, O. Bruyère, T. Cederholm, et al. Writing Group for the European Working Group on Sarcopenia in Older People 2 (EWGSOP2), and the Extended Group for EWGSOP2. Sarcopenia: revised European consensus on definition and diagnosis. Age Ageing 48: 16-31. 2019.

[12] C. Hetz, K. Zhang, R. J. Kaufman. Mechanisms, regulation and functions of the unfolded protein response. Nat Rev Mol Cell Biol. 21(8): 421-438. 2020.

[13] A. Yap, S. Nishiumi, K. I. Yoshida, H. Ashida. Rat L6 myotubes as an in vitro model system to study GLUT4-dependent glucose uptake stimulated by inositol derivatives. Cytotechnology 55(2-3): 103-108. 2007.

[14]E. Lai, T. Teodoro, A. Volchuk. Endoplasmic reticulum stress: signaling the unfolded protein response. Physiology (Bethesda) 22: 193-201. 2007.

[15] K. H. Kim, M. S. Lee. Autophagy-a key player in cellular and body metabolism. Nat Rev Endocrinol. 10(6): 322-337. 2014.

[16] S. Elmore. Apoptosis: a review of programmed cell death. Toxicol Pathol. 35(4): 495-516. 2007. 
[17] J. Chal, O. Pourquié. Making muscle: skeletal myogenesis in vivo and in vitro. Development 144(12): 2104-2122. 2017.

[18]M. C. K. Severinsen, B. K. Pedersen. Muscle-organ crosstalk: The emerging roles of myokines. Endocr Rev. 41(4): 594-609. 2020.

[19] J. H. Kwon, K. M. Moon, K. W. Min. Exercise-Induced Myokines can Explain the Importance of Physical Activity in the Elderly: An Overview. Healthcare (Basel) 8(4): 378. 2020.

[20]F. M. Ashcroft. ATP-sensitive potassium channelopathies: focus on insulin secretion. J Clin Invest. 115(8): 2047-2058. 2005.

[21]F. M. Ashcroft, P. Rorsman. K(ATP) channels and islet hormone secretion: new insights and controversies. Nat Rev Endocrinol. 9(11): 660-669. 2013.

[22] Y. G. Birulina, V. V. Ivanov, E. E. Buyko, E. P. Efremkina, S. V. Smagliy, I. V. Kovalev, et al. Regulation of Vascular Smooth Muscle Contractions in the Model of Metabolic Syndrome. Bull Exp Biol Med. 170(2): 196-199. 2020.

Conflicts of Interest: The authors declare no conflicts of interest.

Contribution of Individual Authors to the Creation of a Scientific Article (Ghostwriting Policy)

Seung-Whan Kim, O-Yu Kwon: Conceptualization, Resources, Supervision, Project administration, Writing Original Draft.

Younghwa Ko, Hyewon Park, Junghae Kim: Methodology, Investigation, Writing - Original Draft.

Kisang Kwon, Kyung-Hee Kang: Review \& Editing, Visualization.

Sources of Funding for Research Presented in a Scientific Article or Scientific Article Itself

The author(s) received a fund (2021. 5. 1. - 2022. 1. 31.) from Chungnam National University, Daejeon, Korea

\section{Creative Commons Attribution License $\mathbf{4 . 0}$} (Attribution 4.0 International, CC BY 4.0)

This article is published under the terms of the Creative Commons Attribution License 4.0 https://creativecommons.org/licenses/by/4.0/deed.en_US 
Table 1. The gene names, GenBank numbers, and sequence of the primers used in the RT-PCR experiments.

\begin{tabular}{|c|c|c|}
\hline Gene & NCBI Reference Sequence & Primer sequence \\
\hline \multirow{2}{*}{$\mathrm{BiP}$} & \multirow{2}{*}{ NM_013083.2 } & F 5'-AGT GGT GGC CAC TAA TGG AG-3' \\
\hline & & R 5'-TCT TTT GTC AGG GGT CGT TC-3' \\
\hline \multirow{2}{*}{ Canx } & \multirow{2}{*}{ NM_172008.2 } & F 5'-GGC ATC TTC ATC CCA GTC AT-3' \\
\hline & & R 5'-CTC CTC TCT GCT CCT CAT GG-3' \\
\hline \multirow{2}{*}{ PDI } & \multirow{2}{*}{ NM_012998.2 } & F 5'-CCA AAG CTG CTG CAA AAC TG-3' \\
\hline & & R 5'-AAA GAG GAC CAC CCC ATC CT-3' \\
\hline \multirow{2}{*}{ ERp29 } & \multirow{2}{*}{ NM_053961.2 } & F 5'-GGC ATC TTC ATC CCA GTC AT-3' \\
\hline & & R 5'-TCC TCT GCA GCT CTT CCT TC-3' \\
\hline \multirow{2}{*}{ PERK } & \multirow{2}{*}{ NM_031599.2 } & F 5'-GGT CTG GTT CCT TGG TTT CA-3' \\
\hline & & R 5'-TTC GCT GGC TGT GTA ACT TG-3' \\
\hline \multirow{2}{*}{ ATF6 } & \multirow{2}{*}{ NM_001107196.1 } & F 5'-CTA GGC CTG GAG GCC AGG TT-3' \\
\hline & & R 5'-ACC CTG GAG TAT GCG GGT TT-3' \\
\hline \multirow{2}{*}{ IRE1 } & \multirow{2}{*}{ NM_001191926.1 } & F 5'-ACC ACC AGT CCA TCG CCA TT-3' \\
\hline & & R 5'-CCA CCC TGG ACG GAA GTT TG-3' \\
\hline \multirow{2}{*}{ XBP1 } & \multirow{2}{*}{ NM_001004210.2 } & F 5'-TCC TCC TGG GTA GAC CTC TG-3' \\
\hline & & R 5'-AAA CAG AGT AGC AGC TCA GA-3' \\
\hline \multirow{2}{*}{$\mathrm{LC} 3 \mathrm{a}$} & \multirow{2}{*}{ NM_199500.2 } & F 5'-GCC TGT CCT GGA TAA GAC CA-3' \\
\hline & & R 5'-GTT CAC CAG CAG GAA GAA GG-3' \\
\hline \multirow{2}{*}{ Beclin } & & F 5'-GTG CTC CTG TGG AAT GGA AT-3' \\
\hline & NIM_00103411/.1 & R 5'-GCT GCA CAC AGT CCA GAA AA-3' \\
\hline & & F 5'-AGG GGC CTT TTT GTT ACA GG-3' \\
\hline Bax & NM_017059.2 & R 5'-GAT CAG CTC GGG CAC TTT AG-3' \\
\hline & & F 5'-TTA CCT CCA CCA GCA GGA AC-3' \\
\hline Вак & NM_053812 & R 5'-ACC ACC TCT CTG TGC AAT CC-3' \\
\hline & & F 5'-AAG CTG TCA CAG AGG GGC TA-3' \\
\hline $\mathrm{Bcl} 2$ & NM_016993 & R 5'-CAG ATG CCG GTT CAG GTA CT-3' \\
\hline & & F 5'-ACC GTG ATT TCC ACC AAG AG-3' \\
\hline Bid & NM_022684 & R 5'-TGG CAA TGT TGT GGA TGA CT-3' \\
\hline MvoD1 & & F 5'-CGA CTG CCT GTC CAG CAT AG-3' \\
\hline IVyор & NM_01/115 & R 5'-GGA CAC TGA GGG GTG GAG TC-3' \\
\hline & & F 5'-CAG CAT CAC GGT GGA GGA TA -3' \\
\hline MyoG & NM_176079 & R 5'-CAC TTA AAA GGC CCC TGC TG -3' \\
\hline & & F 5'-TCA TGC GGA TCA AAC CTC AC-3' \\
\hline Vegfa & NM_001110333 & R 5'-TCT TGC GCT TTC GTT TTT GA -3' \\
\hline & & F 5'-ATT TGA CAT CAC AGC CAC CA -3' \\
\hline BMP7 & NM_001191856.2 & R 5'-GGG AGC TCC ACT TGG ACA AC -3' \\
\hline & & F 5'-GAG GCT GGC ATC CAT GTC TT -3' \\
\hline IL6 & NM_012589.2 & R 5'-CCT CCA GCT CCT CAC ATT CC -3' \\
\hline & & F 5'-ACA GCG ATG ATG CAC TGT CA -3' \\
\hline IL15 & NM_013129.2 & R 5'-GCA TTG GAA GTT GGG GTA GG -3' \\
\hline Anln & NM 031612 & F 5'-CTC TCC TTG ACT GCC GTG TG -3' \\
\hline ApIn & N101_031012 & R 5'-AAA GGC ATG GGT CCC TTA TG-3' \\
\hline & & F 5'-TCC TTC ACA TTT GGC TGT GG -3' \\
\hline Sesn1 & NM_001106396 & R 5'-TTC AAA CCT CGA GGC CAT CT -3' \\
\hline SDF1 & NM 022177 & F 5'-CCT GCC GAT TCT TTG AGA GC -3' \\
\hline SDFI & NM1_0221/1 & R 5'-TTT CGG GTC AAT GCA CAC TT -3' \\
\hline & & F 5'-GAG GGC AAC AAC CTC CTC AC -3' \\
\hline SPARC & NM_012656.1 & R 5'-CGA GTG GTG CAA TGT TCC AT -3' \\
\hline & & F 5'-TGT TCC TGA GGG CTC TGT CA -3' \\
\hline V 1sfatin & NM_1/7928.3 & R 5'-TCT GCC GCT GGA ACA GAA TA -3' \\
\hline & & F 5'-CTT GCA GCT AAG CTC CTG CT -3' \\
\hline Musclin & NM_207612.2 & R 5'-TGG AAC TGG AGA GAC GGT TT -3' \\
\hline & & F 5'-GGG AGC TCC ACT TGG ACA AC-3' \\
\hline Den & NM_024129 & R 5'-ATT TGC CAA TAC CGG ACA GG-3' \\
\hline & & F 5'-GGT GAC TTC ACA CGC CAT AA-3' \\
\hline PPIA & NM_017101.1 & R 5'-CTT CCC AAA GAC CAC ATG CT-3' \\
\hline
\end{tabular}

